\title{
Kanser Hastalarının Bilgi Kaynakları
}

\author{
Information Sources of Cancer Patients
}

Hatice GENCER ${ }^{1}$, Pınar SERÇEKUŞ ${ }^{2}$, Sevgi ÖZKAN ${ }^{3}$

\begin{abstract}
ÖZ
Kanser hastalar1, tedavi konusunda karar vermeleri, fiziksel ve psikolojik ihtiyaçları ile başa çıkmaları için bilgiye ihtiyaç duymaktadır. Doğru bilgiye ulaşılması, tedavi sürecini olumlu yönde etkilemektedir. Kanser hastalarının bilgi edindikleri kaynaklar zaman içinde değişiklik göstermiştir. Hastalar genellikle sağlık çalışanlarından bilgi almak istemekte ve sağlık çalışanlarına güven duymaktadırlar. Ancak son yıllarda bilgi aramak için internet kullanımının arttığı bilinmektedir. Günümüzde internet, hastaların ilk başvurduğu bilgi kaynaklarından biridir. Ancak internet üzerinden alınan bilgiler her zaman doğru olmamaktadır. $\mathrm{Bu}$ nedenle sağlık personelinin hastalığın tüm süreçlerinde birincil bilgi kaynağı olarak yerini korumas1 önemlidir. İnternetin günümüzde s1k kullanılan bilgi kaynağı olduğu da unutulmamalı ve internetten bilgi arayan hastalar güvenli bilgi içeriğine sahip internet kaynaklarına yönlendirilmelidir. $\mathrm{Bu}$ derlemenin amacı kanser hastalarının kullandıkları bilgi kaynaklarının incelenmesidir.
\end{abstract}

Anahtar Kelimeler: Bilgi kaynakları, Kanser, Sağlık

\begin{abstract}
Cancer Patients need some information in order to make decisions about their treatments and meet their physical and physicological needs. Accessing to the reliable information positively affects the treatment process. Information sources of the cancer patients change in time. Generally speaking, patients tend to gather information from the health workers and trust them. However, it is known that the cancer patients have been referring to the Internet as the information source in the recent years. Today, the Internet is regarded as one of the first information sources that is referred by the cancer patients. However, the information gathered from the Internet is not always reliable. Therefore, it is important that the health workers should give information to the cancer patient during the treatment process and that they maintain their place as the primer information source for the patients. On the other hand, it sould be kept in mind that the Internet is a frequently referred source of information and thus the patients who are seeking for information on the Internet should be directed to the Internet sources that have reliable contents. The aim of this compilation was to investigate the information sources of the cancer patients.
\end{abstract}

Keywords: Information resources, Cancer, Health

\footnotetext{
${ }^{1}$ Bilim Uzmanı, Hatice GENCER, Doğum ve Kadın Hastalıkları Hemşireliği, Pamukkale Üniversitesi Sağlık Bilimleri Fakültesi, gencerhatice@gmail.com, ORCID: 0000-0002-4292-6464

${ }^{2}$ Doç. Dr., Pınar SERÇEKUŞ, Doğum ve Kadın Hastalıkları Hemşireliği, Pamukkale Üniversitesi Sağlık Bilimleri Fakültesi, pinarsercekus@gmail.com, ORCID:0000-0002-9326-3453

${ }^{3}$ Prof. Dr., Sevgi ÖZKAN, Doğum ve Kadın Hastalıkları Hemşireliği, Pamukkale Üniversitesi Sağlık Bilimleri Fakültesi, sozkan@ pau.edu.tr, ORCID:0000-0001-8385-210X
} 


\section{GíRiş}

Kanser hastalığı tüm dünyada önemli bir halk sağlığı problemi olarak görülmektedir. ${ }^{1}$ Özellikle gelişmiş toplumlarda, yaşam süresinin uzaması ve sanayileşme sebebiyle artış göstermektedir. ${ }^{2} 2020$ yılında Dünya'da toplam 19,3 milyon yeni kanser vakasi geliştiği ve 10 milyon kansere bağlı ölüm olduğu tahmin edilmektedir. ${ }^{3}$ Türkiye'de ise 2020 y1lında 233834 yeni kanser vakas1 tespit edildiği düşünülmektedir. ${ }^{4} 2019$ yılında kansere bağlı ölüm 77887 kişi olarak tespit edilmiştir. 5

Kanser tanısı almak stresli bir yaşam deneyimidir ve hastalığın doğası gereği hastalar hastalıkları hakkında bilgi almak istemektedir. $^{6}$ Kav ve ark.'nın yaptığ çalışma, eğitim düzeyi yükseldikçe ve yaş azaldıkça bilgi arama davranışlarının arttığını göstermektedir. $^{7}$

Kanser hastaları, hastalık sürecinde bilinçli kararlar alabilmek için tanı, prognoz ve tedavi konularında bilgiye ihtiyaç duymaktadır. ${ }^{8}$ Yapılan bir çalışmada hastaların, spesifik kanser türü $(\% 67,26)$, kemoterapinin yan etkileri ve bunların yönetimi $(\% 63,29)$ ve prognoz $(\% 51,8)$ hakkında bilgi aradıkları tespit edilmiştir. ${ }^{9}$ Farklı bir çalışmada ise bilgi ihtiyacının daha çok tedaviyle ilişkili olduğu saptanmıştır. Hastaların, tanı ve tedavi sırasında hastalığın evresi, tedavi seçenekleri ve tedavinin yan etkileri, tedavi sonrası ise iyileşme süreci ile ilgili bilgiye ihtiyaç duydukları görülmüştür. ${ }^{10}$

Yeterli ve doğru bilgiye ulaşmak, kanser hastalarının sağlık durumlarını olumlu yönde etkilemekte, kansere bağlı oluşan semptomlar ve komplikasyonlarla başa çıkmalarına yardımcı olmaktadır. ${ }^{9}$ Yapılan bir çalışma, bilgi arayan meme kanserli hastalarda fiziksel işlevsellik ve yaşam kalitesinde artış olduğunu göstermektedir. ${ }^{11}$

Kanser hastalarının en sık kullandığ kaynağı sağlık çalışanları olmasına rağmen birçok kanser hastası, ek bir kaynaktan bilgi aramaktadır. Ek bilgi kaynaklarını kullanmada, genç yaş, yüksek gelir, yüksek öğrenim, tamamlayıcı ve alternatif tıp kullanımı gibi faktörler etkili olmaktadır. ${ }^{12}$ Kullanılan kanser bilgi kaynakları yaşanılan topluma göre de farklılık göstermektedir. ${ }^{13,14}$ İran'da yapılan çalışmada en sık kullanılan bilgi kaynağı sırasıyla doktorlar ve televizyon olarak tespit edilmiştir. ${ }^{13}$ Türkiye'de yapılan çalışmada ise en sık başvurulan bilgi kaynakları sırasıyla doktorlar ve internet olarak tespit edilmiştir. ${ }^{14}$ Oysa internette bulunan yanlış bilgiler, yanlış kararlar almaya yol açabilmektedir. ${ }^{15}$ Ülkemizde yapılan bir çalışma, internetten bilgi alan kanser hastalarının kanser ile ilgili aşırı bilgi yüklenmesi yaşadıklarını ortaya koymuştur. ${ }^{16}$ Aşırı bilgi yükü ise kanser hastaları için bilginin etkisiz yönetimine, strese ve belirsizliğe neden olmaktadır. ${ }^{17} \mathrm{Bu}$ nedenle kanser hastalarının bilgi kaynaklarının incelenmesi ve doğru bilgi kaynaklarına yönlendirilmesi önem taşımaktadır. $\mathrm{Bu}$ derlemenin amacı kanser hastalarının kullandıkları bilgi kaynaklarının incelenmesidir.

\section{Kanser Hastalarının Bilgi Kaynakları}

Kanser hastalarının bilgi kaynaklarının; sağlık çalışanları, internet, medya organları ve diğer kanser hastaları olduğu bilinmektedir. ${ }^{13,14}$

\section{Sağlık Çalışanları}

Kanser hastalarının çoğu kanser hakkında bilgilerini yetersiz bulmaktadır. ${ }^{18}$ Yapılan çalışmalar kanser hastaları için en güvenilen ${ }^{8}$ ve en çok başvurulan bilgi kaynağının sağlık çalışanları olduğunu göstermektedir. ${ }^{8-10,14,18}$ Fakat iletişim eksikliği, doktorları rahatsız ettiğini düşünme gibi nedenlerden dolayı hastaların bilgi gereksinimleri sağlık çalışanları tarafından yeterince giderilememektedir. ${ }^{19}$ İletişim, bilgi almanın en etkili yoludur. Hastalara doğru iletişim yöntemleri ile bilgi verilmesinin tedaviye uyumu ve tedavi sonuçlarını olumlu olarak etkilediği bilinmektedir. ${ }^{20}$ Yapılan bir çalışmada meme kanseri tedavisi görmüş kişilerin sağlık personelinin iletişiminden 
memnun olmadığı ve bu kişilerin yaşam kalitesinin daha düşük olduğu bulunmuştur. ${ }^{21}$ Diğer bir çalışma, meme kanserli hastaların en çok tercih ettikleri bilgi kaynağı olan sağlık çalışanlarına yönelik memnuniyet düzeylerinin düşük olduğunu göstermektedir. ${ }^{22}$

Kanser hastalarına verilen bilgiler çeşitli faktörlerin etkisi ile zamanla unutulmaktadır. $\mathrm{Bu}$ nedenle sağlık çalışanları her hasta ziyaretinde hastaların bilgi ihtiyaçlarını yeniden değerlendirmelidir. ${ }^{23}$ Yapılan bir çalışma radyoterapi tedavisi uygulanan kanser hastalarının tedavi sürecinin ilerlemesine rağmen bilgi ihtiyaçlarının yüksek kaldığını ortaya koymaktadır. ${ }^{19}$

Sağlık çalışanları bir bilgi kaynağı olmanın yanında, diğer güvenilir bilgi kaynaklarına yönlendirme konusunda da hastaya rehberlik yapmaktadır. ${ }^{24}$

\section{İnternet}

Dijital çağın getirisi olarak bilgi edinme yolları da zamanla değişmiştir. ${ }^{25}$ İnternet etkili bir bilgi sağlama aracıdır. ${ }^{26}$ Amerika Birleşik Devletleri'ndeki her üç yetişkinden birinin, interneti bir sağllk sorununu teşhis etmek veya öğrenmek için kullandığı tahmin edilmektedir. ${ }^{25}$ Türkiye'de internet \%69,3 oranında sağlıkla ilgili bilgi aramak amaçlı kullanılmaktadır. ${ }^{27}$ İnternetten edinilen bilgiler birçok hastanın tanılarını daha kolay anlamalarını sağlamakta ve tedavi kararlarında etkili olmaktadır. ${ }^{28}$ İnternetten sağlık bilgisinin araştırılması, hastaların bilgileri doktorlar ile tartışmasına ve hastadoktor ilişkisinin gelişmesine de neden olabilmektedir. ${ }^{29}$ İnternetin yaygınlaşmasıyla, kanser hastalarının bilgi kaynağı olarak internet kullanımı da büyük oranda artış göstermiştir. $^{30}$ Ülkemizde 2015 yılında yapılan bir çalışma, kanser hastalarının \%70,8'inin kansere ilişkin bilgileri internetten elde ettiğini göstermektedir. ${ }^{14}$ Farklı bir çalışma ise ilk başvurulan bilgi kaynağının internet olduğunu ortaya koymuştur. İnternet kullanımı özellikle kadınlar, gençler, gelir ve eğitim seviyesi yüksek kişiler arasında daha yaygındır. ${ }^{31}$ Walsh ve ark., eğitim seviyesi yüksek kanser hastalarının tedavi konusunda karar alırken internet ve bilimsel araştırmaları daha fazla kullandıklarını tespit etmiştir. ${ }^{12}$ Chua ve ark.'nın yaptığ 1 çalışma, kanser hastalarının çoğunluğunun interneti tanı ve tedavi seçeneklerini, tedavinin yan etkilerini ve tamamlayıcı ve alternatif tedavi yöntemlerini aramak için kullandığını göstermektedir. ${ }^{32}$ Maloney ve ark.'nın yaptığ 1 çalışmada ise meme kanserli hastaların internet ortamında kanser hakkında bilgiyi en çok kanserle ilgili organizasyonların web sitelerinden elde ettikleri ve en çok yan etkiler ile ilgili bilgi aradıkları tespit edilmiştir. ${ }^{33}$

İnternet bilgi arama ve paylaşma açısından önemli bir alan olsa da internette yer alan bilgiler daima doğru olmamaktadır. ${ }^{14} \mathrm{Bu}$ durum yanlış kararlar almaya yol açabilmektedir. ${ }^{15}$ Kanser tedavisinde alternatif yöntemler sunan web sitelerinin incelendiği bir çalışmada, sitelerin önemli bir bölümünün modern tıbba ve ilaçlara karşı olumsuz bir tutum sergiledikleri, çeşitli videolar, teşekkür mailleri ile yöntemlerinin etkinliğini ispatlamaya çalıştıkları tespit edilmiştir. ${ }^{34}$

İnternette yer alan bilgilerin doğruluğu kadar anlaşılabilir olması da gereklidir. Hazırlanan web sitelerinin hastalara ulaşması için anlaşılabilir ve arama motorları tarafindan kolay bulunabilir olması önemlidir. ${ }^{15}$ Bazı web siteleri zengin ve kaliteli bilgiler içermelerine rağmen özellikle eğitim seviyesi düşük kişiler tarafından anlaşılmaz olabilmektedir. ${ }^{35}$ Hamilton ve ark.'nın yaptığı çalışmada melanom hastalarının çoğunluğunun (\%90) interneti bilgi kaynağı olarak kullandığ kullanilan arama motorunun (\%90) Google olduğu tespit edilmiştir. Hastaların çoğu interneti, faydalı fakat anlaşılması zor bir bilgi kaynağı olarak değerlendirmiştir. ${ }^{28}$ Almanya'da yapılan bir çalışmada, kar amacı gütmeyen web siteleri ve kendi kendine yardım gruplarının oluşturduğu web sitelerinin içerikleri daha kaliteli bulunmuştur. ${ }^{15}$ Çoğu hasta ve yakınları internette yer alan bilgileri doktorların oluşturmasını istemektedirler. ${ }^{26}$ 


\section{Medya (Televizyon, Gazete ve Dergiler)}

Medya tıbbi bilgilerin yaygınlaştırılması için güçlü bir araçtır. $^{32}$ Son yıllarda televizyon kanallarında sağlıkla ilgili programlar, çeşitli derneklerin ve bakanlıkların hazırlamış oldukları kamu spotları kişilerin farkındalık düzeyini arttırmaktadır. $^{30}$ Ülkemizde 2008 yılında yapilan kadınların meme kanseri konusunda bilgi kaynaklarının araştırıldığı bir çalışmada, kadınların en çok televizyon ve gazetelerden bilgi aldıkları tespit edilmiştir. ${ }^{36}$ Özellikle internet kullanımının yaygınlaşmasıyla, yıllar içinde kanser hastalarının bilgi kaynağı olarak medya organlarını kullanma oranı azalmıştır. Medya, genellikle ileri yaşta ve internet kullanım konusunda yetersiz olan kişiler tarafından tercih edilmektedir. ${ }^{26,30}$ 2015 yılında yapılan bir çalışmada, kanser hastalarının sadece \%5'inin televizyonu bilgi aramak için kullandığı tespit edilmiştir. ${ }^{14}$ Shea-Budgell ve ark.'nın 2014 yılında yaptığı çalışmada gazete, dergi, televizyon gibi bilgi kaynaklarının en az başvurulan ve en az güven duyulan kanser bilgi kaynakları olduğu saptanmıştır. ${ }^{8} \mathrm{Bu}$ durum çalışma yapılan toplumlara göre de farklılık göstermektedir. Örneğin; Etiyopya'da 2016 yılında yapılan çalışmada sağlık çalışanlarından sonra en çok tercih edilen kanser bilgi kaynağı, medya organları olarak tespit edilmiştir. Aynı çalışmada interneti bilgi kaynağı olarak kullanan hastaların oranı $\% 5$ olarak tespit edilmiştir. ${ }^{9}$ Yaşanılan toplum, internete erişim olanağı gibi pek çok faktör kanser için kullanılan bilgi kaynaklarında etkili olabilir.

\section{Hasta Kitapçıkları Ve Broşürler}

Kitapçık ve broşürler kanser hastalarını bilgilendirmek amaciyla siklikla kullanılmaktadır. Fiziksel olarak hastalara verilebildiği gibi çevrimiçi dökümanlar olarak da sunulmaktadır. ${ }^{37}$ Kitapçık ve broşürlerin kanser hastalarının en yararlı gördüğü ve en çok tercih ettiği bilgi kaynakları arasında olduğunu gösteren çalışmaların yanında, ${ }^{6,8}$ en az tercih edilen bilgi kaynakları olduğunu gösteren çalışmalar da mevcuttur. ${ }^{13}$ Kitapçık, broşür gibi basılı materyallerin dizaynı etkin kullanımını etkilemektedir. Örneğin içeriğin görsellerle zenginleştirilmesi daha verimli kullanılmasını sağlamaktadır. ${ }^{37}$

\section{Diğer Hastalar ve Hasta Yakınları}

Kanser hastaları özellikle daha sıkıntılı dönemlerinde aile, arkadaş gibi destek kaynaklarından daha çok kendileri ile benzer deneyimler yaşayan kişilerle iletişim kurma eğilimindedirler. ${ }^{38}$ Aynı zamanda kanser tanısı almış diğer hastalardan bilgi edinmektedirler. $\mathrm{Bu}$ durum hastalıkla başa çıkmalarını kolaylaştırmaktadır. ${ }^{30}$ Yapılan bir çalışmada kanser hastalarının diğer hastaları bilgi kaynağı olarak kullanma oranı \%10,1 olarak tespit edilmiştir. ${ }^{26}$ Diğer bir çalışmada ise kanser hastalarının bilgi kaynağı olarak $\% 10,4$ 'ünün arkadaş ve aile üyelerini, \%3,3'ünün ise diğer kanser hastası veya kanserden kurtulan kişileri tercih ettikleri saptanmıştır. ${ }^{32}$

Meme kanserli hastalar üzerinde yapılan çalışma da diğer hastaların bilgi ve deneyimleri en önemli bilgi kaynaklarından biri olarak tespit edilmiştir. ${ }^{13}$ Son yıllarda çevrimiçi destek gruplarının sayılarında artış olmuştur. Meme kanserli hastalar üzerinde yapılan bir çalışma, hastaların çoğunun (91.3) çevrimiçi toplulukları meme kanseri ve tedavisi hakkında bilgi aramak için kullandığını göstermiştir. ${ }^{39}$ Farklı bir çalışmada da ülkemizde kanser ile ilgili blog kullanıcılarının diğer hastalardan blog aracılığıyla bilgi desteği aldığını göstermiştir. ${ }^{40}$ 


\section{SONUÇ VE ÖNERİLER}

Kanser gün geçtikçe sıklığı artan ve yaşamı tehdit eden bir hastalıktır. Hastalara tanı aşamasından başlayarak tedavi, prognoz, tamamlayıcı tedaviler gibi konularda bilgi verilmesi hastalığa ve tedaviye uyumu arttıracaktır. $\mathrm{Bu}$ nedenle sağlık personelinin hastaya, yakınları ile birlikte bilgi vermesi ve birincil bilgi kaynağı olarak yerini koruması büyük önem taşımaktadır. Kanser hastalarında bilgi ihtiyacı ve bilgi edinilen kaynakların zaman içinde değişiklik gösterdiği göz önüne alınmalı ve periyodik aralıklarla hastaların bilgi arama davranışları ve bilgi kaynakları değerlendirmelidir.
İnternetin günümüzde en çok kullanılan bilgi kaynaklarından biri olduğu unutulmamalıdır ve hastalar kaliteli ve güvenli bilgi içeriğine sahip internet bilgi kaynaklarına yönlendirilmelidir. Sağllk personeli, web sitesi tasarlama gibi internette bilgi kaynağı oluşturmada da rol almalıdır. Aynı zamanda hasta ve yakınlarını bilgilendirmek amaçlı hazırlanan broşürlere, bilgi alınabilecek güvenilir internet adresleri eklenebilir. Kanser hastalarının bilgi kaynaklarının incelenmesine yönelik güncel çalışmalar yapılması önerilmektedir.

\section{KAYNAKLAR}

1. Siegel, R.L, Miller K.D. and Jemal, A. (2018). "Cancer Statistics, 2018. CA Cancer". J Clin, 68 (1), 7-30. https://doi.org/10.3322/caac.21442

2. Gürsu, R.U, Kesmezacar, Ö, Karaçetin, D, Mermut, Ö, Ökten, B. ve Güner, Ş.İ. (2012). "İstanbul Eğitim ve Araştırma Hastanesi Onkoloji Birimi: Yeni Kurulan Bir Ünitenin 18 aylık Sonuçları". İstanbul Tıp Derg, 13 (1), 13-18. https://doi.org/10.5505/1304.8503.2012.55264

3. Sung, H, Ferlay, J, Siegel, R.L, Laversanne, M, Soerjomataram, I, Jemal, A. et al. (2021). "Global Cancer Statistics 2020: GLOBOCAN Estimates of Incidence and Mortality Worldwide for 36 Cancers in 185 Countries". CA Cancer J Clin, 71 (3), 209-249. https://doi.org/10.3322/caac.21660

4. World Health Organization International Agency For Research on Cancer. (2020). "Estimated Age-Standardized Incidence Rates (World) in 2020, All Cancers, Both Sexes, All Ages". Erişim Adresi: https://gco.iarc.fr/today/onlineanalysis-map (Erişim Tarihi: 19.04.2021).

5. Türkiye İstatistik Kurumu. (2020). “Ölüm ve Ölüm Nedeni İstatistikleri 2019”. Erişim Adresi: https://data.tuik.gov.tr/Bulten/Index?p=Olum-ve-OlumNedeni-Istatistikleri-2019-33710 (Erișim tarihi: 22 Nisan 2021).

6. Ankem, K. (2006). "Use of İnformation Sources by Cancer Patients: Results of a Systematic Review of the Research Literature". Information Research, 11 (3), 254.

7. Kav, S, Tokdemir, G, Tasdemir, R, Yalili, A. and Dinc, D. (2012). "Patients with Cancer and their Relatives Beliefs, İnformation Needs and İnformation-Seeking Behavior about Cancer and Treatment". Asian Pac J Cancer Prev, 13 (12), 6027-6032. https://doi.org/10.7314/APJCP.2012.13.12.60278

8. Shea-Budgell, M, Kostaras, X, Myhill, K. and Hagen, N. (2014). "Information Needs and Sources of İnformation for Patients During Cancer Follow-up". Curr Oncol, 21 (4), 165174. http://dx.doi.org/10.3747/co.21.1932

9. Mekuria, A.B, Erku, D.A. and Belachew, S.A. (2016) "Preferred İnformation Sources and Needs of Cancer Patients on Disease Symptoms and Management: A Cross-Sectional Study". Patient Prefer Adherence, 10, 1991-1997. https://doi.org/10.2147/PPA.S116463
10. Rutten, L.J, Arora, N.K, Bakos, A.D, Aziz, N. and Rowland, J. (2005). "Information Needs and Sources of İnformation Among Cancer Patients: A Systematic Review of Research (1980-2003)". Patient Educ Couns, 57 (3), 250-261. https://doi.org/10.1016/j.pec.2004.06.006

11. Ransom, S, Jacobsen, P.B, Schmidt, J.E. and Andrykowski, M.A. (2005). "Relationship of Problem-Focused Coping Strategies to Changes in Quality of Life Following Treatment for early stage breast cancer". J Pain Symptom Manage, 30 (3), 243-253. https://doi.org/10.1016/j.jpainsymman.2005. 03.013

12. Walsh, M.C, Trentham-Dietz, A, Schroepfer, TA, Reding, D.J, Campbell, B, Foote, M.L. et al. (2010). "Cancer İnformation Sources Used by Patients to İnform and İnfluence Treatment Decisions". J Health Commun, 15 (4), 445-463. https://doi. org/10.1080/10810731003753109

13. Kimiafar, K, Sarbaz, M, Shahid Sales, S, Esmaeili, M. and Javame Ghazvini, Z. (2016). 'Breast Cancer Patients' İnformation Needs and İnformation-Seeking Behavior in a Developing Country". Breast, 28, 156-160. https://doi.org/10.1016/j.breast.2016.05.011

14. Başkale, H.A, Serçekuş, P. ve Günüșen, N.P. (2015). "Kanser Hastalarının Bilgi Kaynakları, Bilgi Gereksinimleri ve Sağlık Personelinden Beklentilerinin İncelenmesi”. Psikiyatri Hemșireliği Dergisi, 6 (2), 65-70. https://doi.org/10.5505/phd. 2015.49091

15.Liebl, P, Seilacher, E, Koester, M-J, Stellamanns, J, Zell, J. and Hübner, J. (2015). "What Cancer Patients Find in the Internet: The Visibility of Evidence-Based Patient İnformation Analysis of İnformation on German Websites". Oncol Res Treat, 38 (5), 212-218. https://doi.org/10.1159/000381739

16. Serçekuş, P, Gencer, H. ve Özkan, S. (2020). "Finding Useful Cancer Information May Reduce Cancer Information Overload For Internet Users". Health Info Libr J, 37 (4), 319328. https://doi.org/10.1111/hir.12325

17. Kim, K, Lustria, M.L, Burke, D. and Kwon, N. (2007). "Predictors of Cancer Information Overload: Findings From a National Survey". Information Research, 12 (4), 12-14.

18. Chen, X. and Siu, L.L. (2001). "Impact of the Media and the Internet on Oncology: Survey of Cancer Patients and 
Oncologists in Canada". J Clin Oncol, 19 (23), 4291-4297. https://doi.org/10.1200/JCO.2001.19.23.4291

19. Douma, K.F, Koning, C.C, Zandbelt, L.C, de Haes, H.C. and Smets, E.M. (2012). "Do Patients' Information Needs Decrease Over the Course of Radiotherapy?". Support Care Cancer, 20 (9), 2167-2176. https://doi.org/10.1007/s00520011-1328-0

20. Sheppard, V.B, Adams, I.F, Lamdan, R. and Taylor, K.L. (2011). "The Role of Patient-Provider Communication for Black Women Making Decisions About Breast Cancer Treatment". Psychooncology, 20 (12), 1309-1316. https://doi.org/10.1002/pon.1852

21. Miyashita, M, Ohno, S, Kataoka, A, Tokunaga, E, Masuda, N, Shien, T. et al. (2015). "Unmet Information Needs and Quality of Life in Young Breast Cancer Survivors in Japan". Cancer Nursing, 38 (6), E1-E11. https://doi.org/10.1097/ncc.0000000 000000201

22.Li, P.W, So, W.K, Fong, D.Y, Lui, L.Y, Lo, J.C. and Lau, S.F. (2011). "The Information Needs of Breast Cancer Patients in Hong Kong and Their Levels of Satisfaction With the Provision of Information". Cancer Nursing, 34 (1), 49-57. https://doi.org/10.1097/NCC.0b013e3181ef77a0

23. Jansen, J, Butow, P.N, van Weert, J.C, van Dulmen, S, Devine, R.J, Heeren, T.J. et al. (2008). "Does Age Really Matter? Recall of Information Presented to Newly Referred Patients With Cancer". Journal of Clinical Oncology, 26 (33), 5450-5457. https://doi.org/10.1200/JCO.2007.15.2322

24.Diaz, J.A, Sciamanna, C.N, Evangelou, E, Stamp, M.J. and Ferguson, T. (2005). "Brief Report: What Types of Internet Guidance Do Patients Want From Their Physicians?" J Gen Intern Med, 20 (8), 683-685. https://doi.org/10.1111/j.15251497.2005.0115.x

25. Jacobs, W, Amuta, A.O. and Jeon, K.C. (2017). "Health Information Seeking in the Digital Age: An Analysis of Health Information Seeking Behavior Among US Adults". Cogent Soc Sci, 3 (1), 1302785. https://doi.org/10.1080/233 11886.2017.1302785

26.James, N, Daniels, H, Rahman, R, McConkey, C, Derry, J. and Young, A. (2007). "A Study of Information Seeking by Cancer Patients and Their Carers". Clin Oncol, 19 (5), 356362. https://doi.org/10.1016/j.clon.2007.02.005

27. Türkiye İstatistik Kurumu. (2020). "Türkiye İstatistik Kurumu Hanehalkı Bilişim Teknolojileri Kullanım Araştırması". Erişim Adresi: http://www.tuik.gov.tr/PreTablo.do?alt_id= 1028 (Erişim tarihi 16 Şubat 2020).

28. Hamilton, S.N, Scali, E.P, Yu, I, Gusnowski, E. and Ingledew, P.A. (2015). "Sifting Through It All: Characterizing Melanoma Patients' Utilization of the Internet As an Information Source". J Cancer Educ, 30 (3), 580-584. https://doi.org/10.1007/s13187-014-0711-1

29. Tan, S.S. and Goonawardene, N. (2017). "Internet Health Information Seeking and the Patient-Physician Relationship: A Systematic Review". J Med Internet Res, 19 (1), 9. https://doi.org/10.2196/jmir.5729

30. Yıldırım, S. (2015). Kanser Tanısı İle İzlenen Hastaların Yakınlarının Kanser İle İlgili Başvurdukları Bilgi Kaynaklarının Değerlendirilmesi. (Uzmanlık Tezi), Dokuz Eylül Üniversitesi, Tıp Fakültesi, İç Hastalıkları ABD, İzmir.

31.Hesse, B.W, Nelson, D.E, Kreps, G.L, Croyle, R.T, Arora, N.K, Rimer, B.K. et al. (2005). "Trust and Sources of Health Information: The Impact of the Internet and Its Implications for Health Care Providers: Findings From the First Health Information National Trends Survey". Arch Intern Med, 165 (22), 2618-2624. https://doi.org/10.1001/archinte.165.22.2618

32. Chua, G.P, Tan, H.K. and Gandhi, M. (2018). "Information Sources and Online Information Seeking Behaviours of
Cancer Patients in Singapore". Ecancermedicalscience, 12, 880. https://doi.org/10.3332/ecancer.2018.880

33. Maloney, E.K, D'Agostino, T.A, Heerdt, A, Dickler, M, Li, Y, Ostroff, J.S. et al. (2015). "Sources and Types of Online Information That Breast Cancer Patients Read and Discuss With Their Doctors". Palliat Support Care, 13 (2), 107-114. https://doi.org/10.1017/S1478951513000862

34. Tekin, A, Esin, K. ve Yazıcı, S.Ö. (2012). "Kanserle İlgili Alternatif Tıp İçerikli Web Sitelerinin İçerik Analizi". Mehmet Akif Ersoy Üniversitesi Sosyal Bilimler Enstitüsü Dergisi, 0 (6), 14-34.

35. Alsaiari, A, Joury, A, Aljuaid, M, Wazzan, M. and Pines, J.M. (2017). "The content and Quality of Health Information on the Internet For Patients and Families on Adult Kidney Cancer". J Cancer Educ, 32 (4), 878-884. https://doi.org/10.1007/s13187016-1039-9.

36. Özaydın, A.N, Güllüoğlu, B.M, Ünalan, P.C, Gorpe, S, Öner, B.R. ve Özmen, V. (2009). "Bahçeşehir'de Oturan Kadınların Meme Kanseri Bilgi Düzeyleri, Bilgi Kaynakları ve Meme Sağlığı ile İlgili Uygulamaları”. Meme Sağlığı Dergisi, 5 (4), 214-224.

37.King, A.J. (2015). "A Content Analysis of Visual Cancer Information: Prevalence and Use of Photographs and Illustrations in Printed Health Materials". Health Communication, $30 \quad$ (7), 722-731. https://doi.org/ 10.1080/10410236.2013.878778

38.Zhang, S, Bantum, E.O, Owen, J, Bakken, S. and Elhadad, N. (2016). "Online Cancer Communities As Informatics Intervention For Social Support: Conceptualization, Characterization, and Impact”. J Am Med Inform Assoc, 24 (2), 451-459. https://doi.org/10.1093/jamia/ocw093

39. Bender, J.L, Katz, J, Ferris, L.E. and Jadad, A.R. (2013). "What is the Role of Online Support From the Perspective of Facilitators of Face-to-Face Support Groups? A Multi-Method Study of the Use of Breast Cancer Online Communities", $\begin{array}{llll}\text { Patient Educ Couns, } 93 & \text { (3), 472-479. }\end{array}$ https://doi.org/10.1016/j.pec.2013.07.009

40. Serçekuş, P. and Başkale, H. (2015). "Living and Coping With Cancer: Experiences of Cancer Blog Users in Turkey". Holist Nurs Pract, 29 (3), 144-150. https://doi.org/10.1097/hnp.00 00000000000082 